\title{
Magnetophotoluminescence study of the influence of substrate orientation and growth interruption on the electronic properties of InAs/GaAs quantum dots
}

\author{
S. Godefroo, J. Maes, M. Hayne, and V. V. Moshchalkov \\ Pulsed Field Group, Laboratory of Solid State Physics and Magnetism, K.U. Leuven Celestijnenlaan \\ 200 D, B-3001 Leuven, Belgium \\ M. Henini, F. Pulizzi, A. Patanè, and L. Eaves \\ School of Physics and Astronomy, University of Nottingham, Nottingham NG7 2RD, United Kingdom
}

(Received 25 March 2004; accepted 10 May 2004)

\begin{abstract}
We have used photoluminescence in pulsed $(\leqslant 50 \mathrm{~T})$ and dc $(\leqslant 12 \mathrm{~T})$ magnetic fields to investigate the influence of substrate orientation and growth interruption (GI) on the electronic properties of InAs/GaAs quantum dots, grown by molecular beam epitaxy at $480{ }^{\circ} \mathrm{C}$. Dot formation is very efficient on the (100) substrate: electronic confinement is already strong without GI and no significant change in confinement is observed with GI. On the contrary, for the (311)B substrate strong confinement of the charges only occurs after a GI is introduced. When longer GIs are applied the dots become higher. (C) 2004 American Institute of Physics. [DOI: 10.1063/1.1767972]
\end{abstract}

\section{INTRODUCTION}

Quantum dot (QD) lasers promise some advantages over quantum well and quantum wire lasers: a lower threshold current density, better temperature stability, higher optical gain, and no chirp. ${ }^{1}$ To fabricate QD lasers, it is very important to find methods to reproducibly grow QDs with a high degree of size uniformity. Therefore, the dependence of the properties of the dots on growth conditions such as substrate orientation, and growth interruption, and growth temperature is studied by many groups. ${ }^{2-6}$

Tilted substrates, in which the surface is other than (100), allow us to make use of extra parameters in the search for better technological structures because the natural roughness on high index substrates influences the dot formation and the electronic properties of the dots, such that the photoluminescence (PL) energy, the PL intensity, or the PL linewidth can change according to the substrate orientation. ${ }^{2,3,5}$ A study of the QD growth on (n11)B substrates has shown that (311)B has the optimum degree of QD ordering and the greatest homogeneity in their size. ${ }^{5}$ The electronic properties of the dots are also influenced by introducing a growth interruption (GI) before deposition of the capping layer that fixes the dots at their position. Atom migration during GI may change the size, shape, and composition of the dots, and thus their electronic properties.

Here, magnetophotoluminescence is used to obtain more insight into the structural and electronic properties of InAs/GaAs quantum dots grown on (100) and (311)B substrates at a growth temperature of $480{ }^{\circ} \mathrm{C}$. Our data indicate that for the (100) substrate the dots rapidly form an equilibrium morphology. In contrast, for the (311)B substrate, a large decrease in the PL shift for structures with GI compared with those without GI shows that strong confinement of the charges only occurs after a GI is introduced.

\section{EXPERIMENTAL DETAILS}

The self-assembled InAs/GaAs quantum dots were grown at the University of Nottingham by molecular beam epitaxy (MBE). A semi-insulating GaAs substrate was covered with a $500 \mathrm{~nm}$ thick GaAs layer. On this, a 1.8 monolayer (ML) thick InAs layer was deposited at a temperature of $480{ }^{\circ} \mathrm{C}$ and with a growth rate of $0.0666 \mathrm{ML} \mathrm{s}^{-1}$. After the desired GI, the dots were capped with a $25.2 \mathrm{~nm} \mathrm{GaAs}$ layer. The GI is varied between $0,40,80$, and $120 \mathrm{~s}$. Two different sets of samples are studied. Set A consists of samples with a (100) oriented GaAs substrate, whereas the samples in set $B$ have a (311)B oriented GaAs substrate. We refer to each sample by a letter indicating the substrate orientation followed by a number, which is the GI in seconds.

PL measurements were performed at $4.2 \mathrm{~K}$ in pulsed magnetic fields up to $50 \mathrm{~T}$ and at $10 \mathrm{~K}$ in dc fields up to $12 \mathrm{~T}$. In pulsed fields, the magnetic field $B$ was applied parallel (\|) or perpendicular $(\perp)$ to the growth direction $z$, while for the dc measurements the field was only applied parallel to $z$. Light from a frequency doubled neodymium:yttriumaluminum-garnet laser, with a wavelength of $532 \mathrm{~nm}$ and a maximum power output of $100 \mathrm{~mW}$, excited the sample, via an optical fibre with a core diameter of $200 \mu \mathrm{m}$. The laser intensity was always low enough to excite only the ground state exciton. Six surrounding optical fibres collected the PL signal and transferred it to a liquid-nitrogen cooled InGaAs detector via a $30 \mathrm{~cm}$ spectrometer. In pulsed fields up to four spectra were taken during a $20 \mathrm{~ms}$ magnetic field pulse with an integration time of typically $0.5 \mathrm{~ms}$. It should be noted that in contrast with pulsed-field experiments, in dc fields the photon integration time can be arbitrarily extended.

From the PL spectra in the absence of a magnetic field, the PL energy and PL linewidth are obtained. The PL energy is taken to be the center of mass of the peak $\left(E_{\mathrm{CM}}\right)$ and the PL linewidth is the full width at half maximum of the peak. For pulsed-field measurements, careful analysis of the field dependence of the center of mass of the peak can provide two electronic properties: Where possible, the exciton radius $\sqrt{\left\langle\rho^{2}\right\rangle}$ and the effective mass $\mu$ are obtained using a model for the shift of the center of mass of the PL peak in field that is parabolic at low and linear at high magnetic fields: ${ }^{7}$ 


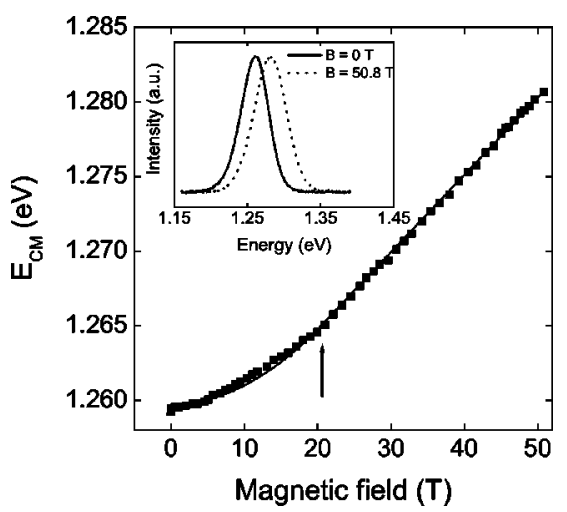

FIG. 1. The center of mass of the PL peak $\left(E_{\mathrm{CM}}\right)$ as a function of magnetic field for pulsed-field measurements of sample A00 for $B \| z$. The squares represent the data while the line shows the fit to the data. The arrow indicates the crossover field between the low and high field regimes. The inset shows the normalized luminescence spectra for structure A00 without field (full line) and for a magnetic field of $50.8 \mathrm{~T}$ parallel to the growth direction (dashed line)

$$
\begin{aligned}
& \Delta E_{\mathrm{CM}}=\frac{e^{2}\left\langle\rho^{2}\right\rangle}{8 \mu} B^{2} \quad \text { for } \quad B<\frac{2 \hbar}{e\left\langle\rho^{2}\right\rangle}, \\
& \Delta E_{\mathrm{CM}}=-\frac{\hbar^{2}}{2 \mu\left\langle\rho^{2}\right\rangle}+\frac{\hbar e B}{2 \mu} \quad \text { for } B>\frac{2 \hbar}{e\left\langle\rho^{2}\right\rangle} .
\end{aligned}
$$

The transition between the two regimes occurs when the magnetic length $l_{B}=(\hbar / e B)^{1 / 2}$ equals $\left(\left\langle\rho^{2}\right\rangle / 2\right)^{1 / 2}$. An example is given in Fig. 1 where the model (solid line) is applied to the A00 sample data for $B \|_{z}$ (squares). The transition occurs at $20.6 \mathrm{~T}$ and is indicated by an arrow. Here, the exciton radius and effective mass are determined as $8.0 \mathrm{~nm}$ and $0.11 m_{0}$ respectively, with $m_{0}$ being the free electron mass. The exciton radius as obtained by this technique is the smallest extent of the exciton in the plane perpendicular to the field direction. ${ }^{8}$ The effective exciton mass $\mu$ is determined by the band structure of the material, which is changed by the strain in the dots caused by the difference in lattice constant between InAs and GaAs. This results in an effective exciton mass which is larger in QDs in comparison with that of bulk material. ${ }^{9}$ The $\mu$ value is obtained from the slope of the linear part of the energy shift [Eq. (1b)].

Information about charge confinement can also be inferred from the magnitude of the PL shift, even when the data do not conform to Eqs. (1a) and (1b). Therefore, we define $\Delta E_{\mathrm{CM}}$ as the PL shift of the center of mass of the peak between $0 \mathrm{~T}$ and $50 \mathrm{~T}$. It should be noted that this is not necessarily the maximum energy shift because some samples show a decrease in $E_{\mathrm{CM}}$ around $5 \mathrm{~T}$, such that the minimum $E_{\mathrm{CM}}$ is not at $B=0 \mathrm{~T}$. The origin of these unexpected decreases in $E_{\mathrm{CM}}$ is presently unknown, but their presence has been verified in dc fields for $B \|_{z}$. In Fig. 2 the pulsed-field data of B40 for $B \|_{z}$ are shown, whereas the inset shows the dc data. The latter allows a more detailed analysis at low fields and a decrease in $E_{\mathrm{CM}}$ of $0.8 \mathrm{meV}$ between 0 and $6 \mathrm{~T}$ is derived. The offset in PL energy of $\sim 10 \mathrm{meV}$ between the data in the main part of the figure and that in the inset is caused by illumination of different parts of the sample in the two experiments.

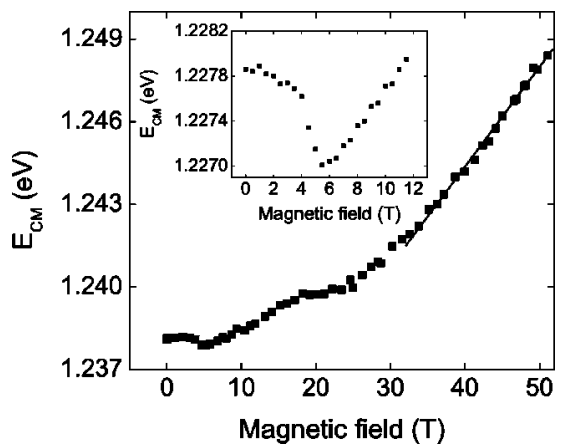

FIG. 2. The center of mass of the PL peak $\left(E_{\mathrm{CM}}\right)$ as a function of magnetic field for pulsed-field measurements and dc measurements (inset) of sample B40 for $B \|_{z}$. A decrease in $E_{\mathrm{CM}}$ of $0.8 \mathrm{meV}$ between 0 and $6 \mathrm{~T}$ is observed in the data obtained by dc fields. The solid line represents the linear fit.

\section{RESULTS}

\section{A. (100) substrate}

Figure 3(a) shows the PL spectra for different GIs on the (100) substrate, while in Fig. 3(c) the PL energy is plotted as
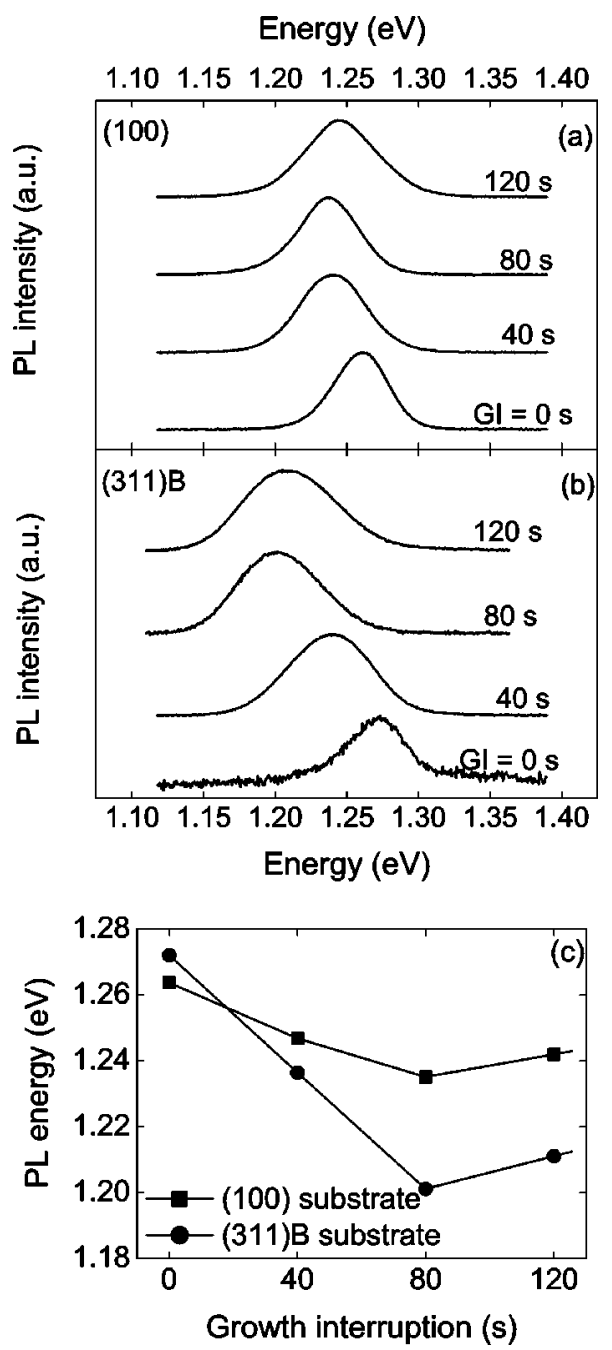

FIG. 3. PL spectra of InAs QDs grown on (100) (a) and (311)B (b) substrates for different growth interruptions (GI) in the absence of a magnetic field. The PL energy as a function of GI is shown in (c). The data for the (100) and (311)B substrates are represented by squares and circles, respectively. 
TABLE I. The PL linewidth, the exciton radius $\sqrt{\left\langle\rho^{2}\right\rangle}$ and the effective exciton mass $\mu$ for $B \|_{z}$ for structures with the (100) orientation.

\begin{tabular}{cccc}
\hline \hline & PL linewidth $(\mathrm{meV})$ & $\sqrt{\left\langle\rho^{2}\right\rangle}(\mathrm{nm})$ & $\mu\left(m_{0}\right)$ \\
\hline A00 & 45.4 & 8.0 & 0.11 \\
A40 & 52.7 & 6.8 & 0.18 \\
A80 & 47.7 & 8.1 & 0.12 \\
A120 & 56.2 & 6.5 & 0.11 \\
\hline \hline
\end{tabular}

a function of GI for both substrates. The PL energy is dependent on the size of the dots, the strain, the quantum dot composition, ${ }^{10}$ etc., and is shifted to higher energies $(\sim 1.25 \mathrm{eV})$ in comparison with that of bulk InAs $(0.4 \mathrm{eV}$, Ref. 11), mainly due to strong charge confinement. For the (100) substrate, the variations in PL energy are relatively small: there is a redshift of $28.6 \mathrm{meV}$ between B00 and B80, and a small blueshift between B80 and B120. Comparable results were obtained in Ref. 4. It should be noted, however, that they observed a $70 \mathrm{meV}$ redshift for a lower growth temperature of $460{ }^{\circ} \mathrm{C}$ while for a higher growth temperature of $500{ }^{\circ} \mathrm{C}$, again no significant changes were observed. The PL linewidth is a measure of the uniformity of the dots, e.g., in chemical composition, size of the dots, etc. For the (100) substrate we see a small increase in linewidth between 0 and $40 \mathrm{~s}$ GI, but beyond this, no clear trend is observed (Table I).

We now turn to the behavior of the QDs in magnetic field. The inset of Fig. 1 shows the normalized luminescence spectra of structure A00 for zero field and a magnetic field of $50.8 \mathrm{~T}$ parallel to the growth direction. There is an obvious blueshift of the luminescence peak of $20.5 \mathrm{meV}$, which is smaller than the linewidths of the peaks $(45.4 \mathrm{meV}$ and $54.8 \mathrm{meV}$ for $0 \mathrm{~T}$ and $50.8 \mathrm{~T}$, respectively). For the field perpendicular to the growth direction, $\Delta E_{\mathrm{CM}}$ is only $7.8 \mathrm{meV}$ and this shows the importance of an accurate determination of the center of mass. The PL shift $\Delta E_{\mathrm{CM}}$ is a measure of the spatial confinement of the charge carriers in the plane perpendicular to the magnetic field: for a constant effective mass, a stronger spatial confinement of the carriers corresponds to a smaller $\Delta E_{\mathrm{CM}}$ [Eqs. (1a) and (1b)]. The values of the PL shift for the (100) substrate orientation are shown in Fig. 4(a) for both field directions. For $B \perp z, \Delta E_{\mathrm{CM}}$ does not change significantly while for $B \| z$, the value of $\Delta E_{\mathrm{CM}}$ for A40 deviates from the values of the other samples. Such a deviation is also seen in the effective mass for $B \|_{z}$ (Table I). There is an increase in $\mu$ between A00 and A40, but the increase is not persistent: the effective mass for A80 returns almost to the value for A00. Since the PL shift is related to $\left\langle\rho^{2}\right\rangle$ and inversely proportional to $\mu$ [Eqs. (1a) and (1b)], the product of $\Delta E_{\mathrm{CM}}$ and $\mu / m_{0}$ is only dependent on $\left\langle\rho^{2}\right\rangle^{1 / 2}$ and independent of $\mu$. This product shows no discontinuity for A40 [Fig. 4(c)], implying that for this sample the charge confinement is similar to the other samples, but that there is an anomaly in the exciton mass.

Summarizing the data for the (100) substrate, the effective mass (Table I), PL shift [Fig. 4(a)], and the PL energy [Fig. 3(c)] indicate that for the (100) substrate the dots do not

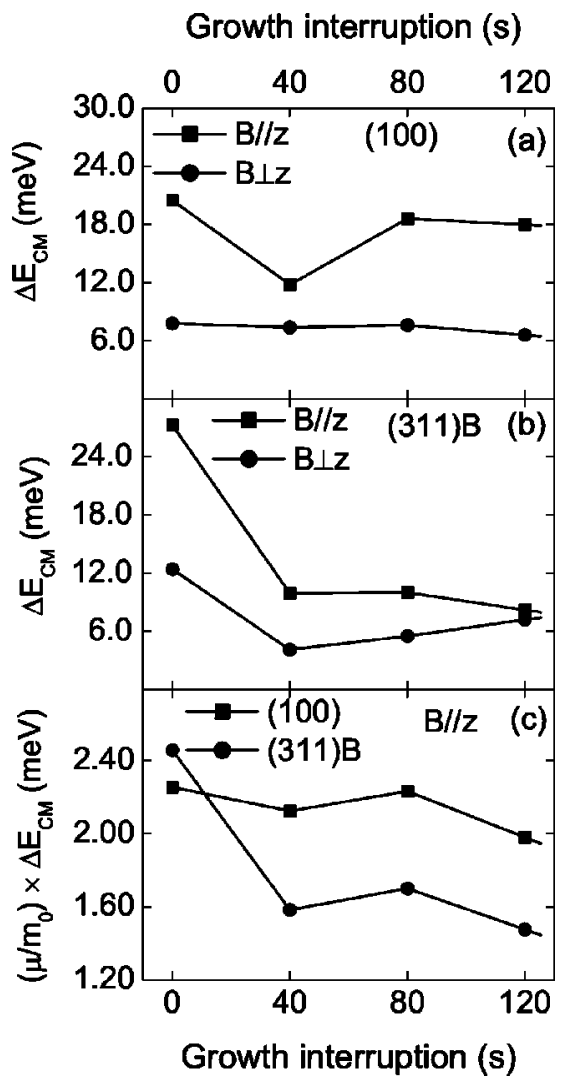

FIG. 4. The PL shift $\left(\Delta E_{\mathrm{CM}}\right)$ as function of the growth interruption for samples with the (100) (a) and (311)B (b) substrate orientations for both $B \|_{z}$ (squares) and $B \perp z$ (circles). The product of the effective mass $\mu / m_{0}$ and PL shift for $B \| z$, also as a function of growth interruption, is shown in (c) for the (100) (squares) and (311)B (circles) substrate orientations. This product is a measure of the exciton extent. The lines always join the points.

significantly evolve during the GI, i.e., there is little diffusion of the atoms during the GI and the dots very rapidly form an equilibrium morphology.

\section{B. (311)B substrate}

The PL spectra for different growth interruptions on the (311)B substrate are shown in Fig. 3(b). As can be seen from the PL spectra and Table II, the linewidth shows a large systematic increase revealing an increasing nonuniformity of the dots. In contrast with the (100) substrate, the stronger change in PL energy with GI on the (311)B substrate [Fig. 3(c)] seems to provide tunability of the emission wavelength: there is a redshift of $70.8 \mathrm{meV}$ between B00 and B80, and a blueshift of $9.9 \mathrm{meV}$ between B80 and B120. This confirms the measurements of Ochoa et $a l^{4}$ and indicates that the

TABLE II. The PL linewidth, the exciton radius $\sqrt{\left\langle\rho^{2}\right\rangle}$, and the effective exciton mass $\mu$ for $B \|_{z}$ for structures with the (311)B orientation. Missing data indicate that for that particular sample the center of mass of the PL peak does not obey the parabolic and linear behavior as a function of $B$.

\begin{tabular}{cccc}
\hline \hline & PL linewidth $(\mathrm{meV})$ & $\sqrt{\left\langle\rho^{2}\right\rangle}(\mathrm{nm})$ & $\mu\left(m_{0}\right)$ \\
\hline B00 & 54.0 & 9.4 & 0.09 \\
B40 & 66.7 & & 0.16 \\
B80 & 68.1 & $<5.8$ & 0.17 \\
B120 & 74.8 & & 0.18 \\
\hline \hline
\end{tabular}




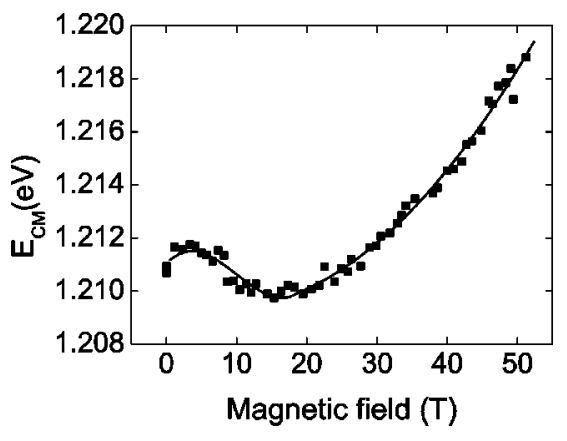

FIG. 5. The center of mass of the PL peak $\left(E_{\mathrm{CM}}\right)$ as a function of magnetic field for pulsed-field measurements of structure B120 for $B \perp z$, where neither a clear parabolic behavior for low fields nor a linear behavior for high fields can be seen. The line is a guide to the eye.

properties of the dots keep changing during GI at $480{ }^{\circ} \mathrm{C}$ for the (311)B substrate. However, in Ref. 4 no significant changes were observed for growth on this substrate at temperatures of $460{ }^{\circ} \mathrm{C}$ and $500{ }^{\circ} \mathrm{C}$.

For $B \perp z$, the energy shift is often found to deviate from the parabolic and linear behavior ${ }^{7}$ as shown in Fig. 5 for B120 (the line is a guide to the eye). At present we have no satisfactory explanation for this behavior. For the (311)B set, a deviation from the parabolic low field behavior is even seen for $B \| z$ with finite GI. Figure 2 gives the example for B40 and $B \|_{z}$ : a clear linear behavior for high magnetic fields can be noticed while for low magnetic fields the data deviate from the expected parabolic behavior. Specifically, $E_{\mathrm{CM}}$ decreases at very low fields before increasing in the usual way. For this reason, the parabolic and linear model cannot be applied to analyze the data, but $\mu$ can nevertheless be obtained from the slope of the linear part at high fields, assuming that the energy shift is determined by the lowest Landau level. For B40 the linear part starts around $32 \mathrm{~T}$ and a linear fit (straight line in Fig. 2) is used to determine the slope as $0.362 \mathrm{meV} / \mathrm{T}$, giving an effective mass of $0.16 \mathrm{~m}_{0}$. Due to deviation from parabolic behavior at low field for $B \| z$, only one value for the exciton radius is included in Table II, namely, for B00. For B80, $E_{\mathrm{CM}}$ obeys the parabolic and linear behavior, but the transition between the two regimes only occurs at $40 \mathrm{~T}$. Due to the high value of this transition field, the exciton radius, determined as $5.8 \mathrm{~nm}$, has to be considered as an upper limit.

Figure 4(b) shows the values of the PL shift for the (311)B substrate for both field directions. Remarkable are the decreases in $\Delta E_{\mathrm{CM}}$ between 0 and $40 \mathrm{~s}$ GI for both $B \|_{z}$ and $B \perp z$. Since we do not know when these decreases exactly happen (they may happen anywhere between 0 and $40 \mathrm{~s}$ ), the lines between the B00 and B40 samples for both field directions are just indicative and connect the data points of 0 and $40 \mathrm{~s}$. After the decrease, $\Delta E_{\mathrm{CM}}$ stays constant for $B \| z$ while for $B \perp z$ an increase is observed. This indicates that after $40 \mathrm{~s}$ GI the charge carriers are less vertically spatial confined while the confinement in the plane of the sample does not change.

Remarkable in Table II is the large increase in effective mass between structures B00 and B40. The mass is independent of the length of the GI for larger GIs, but between B00 and B40 the increase in effective mass amounts to $80 \%$. For the explanation of this large increase in $\mu$, we refer to our earlier work, ${ }^{2}$ where for InAs QDs grown on a (311)B GaAs substrate, it was found that the exciton is not completely confined in the dot for InAs depositions below 1.9 ML, and that the dots are not yet fully developed. In this study, the sample without GI is nominally identical to the $1.8 \mathrm{ML}$ sample from Ref. 2 and behaves similarly concerning the PL shift in both field directions. One can thus conclude that also in our sample the excitons are not completely confined to the QD. Furthermore, the decrease in PL shift between B00 and $\mathrm{B} 40$ is stronger than expected from the change in $\mu$ alone: the PL shift is inversely proportional to the effective mass as shown in Eqs. (1a) and (1b). The increase of $80 \%$ in $\mu$ should thus lead to $\Delta E_{\mathrm{CM}} \approx 15.2 \mathrm{meV}$ for $\mathrm{B} 40$. However, the measured value is only $9.9 \mathrm{meV}$. This extra decrease indicates that the exciton wave functions are shrunk not only due to an increase in $\mu$ but also due to a stronger lateral spatial confinement. So, with growth interruption, the QDs develop so that the wave functions are more efficiently confined in the QD. We speculate that the increase in effective mass is the result of a relative increase in electron confinement compared to hole confinement as the dots evolve. The decrease in $\sqrt{\left\langle\rho^{2}\right\rangle}$ between $9.4 \mathrm{~nm}$ for B00 and the upper limit of $5.8 \mathrm{~nm}$ for B80 confirms the increase in lateral confinement provided by the GI.

Although we have no values for the effective mass for (311)B for $B \perp z$, we assume that it follows the same trend as for $B \| z$, i.e., the effective mass is constant after $40 \mathrm{~s}$ GI. If this is the case, we can compare the PL shifts for structures $\mathrm{B} 40, \mathrm{~B} 80$, and $\mathrm{B} 120$ purely in terms of exciton confinement for $B \perp z: \Delta E_{\mathrm{CM}}$ increases with GI so the charge carriers are less spatially confined in the growth direction, corresponding to an increasing extent of the exciton in the growth direction. For the structure with $120 \mathrm{~s}$ GI, we thus find a large extent of the exciton in the growth direction, and this is corroborated by a cross sectional TEM image of a simple QD in sample B120 with a diameter larger than $30 \mathrm{~nm}$ and a height around $12 \mathrm{~nm}^{4}{ }^{4}$ A diameter of $30 \mathrm{~nm}$ is not exceptional, but the height is unusually large, a few nanometres being more typical. ${ }^{3}$ This evolution of the dots may be caused by the diffusion of In and Ga atoms during the GI. This could also lead to less uniform dots and thus to the increase in linewidth with GI that was observed. We note that the increase in PL linewidth is the largest between B00 and B40, and it corresponds with the most substantial changes in the excitonic properties.

For structures on a (311)B substrate, we can conclude that the dots are not yet in an equilibrium state when they are immediately capped and that, in comparison with the (100) substrate, there is more diffusion of In and Ga atoms during the GI. In the following section we will discuss this in more detail.

\section{DISCUSSION}

The data from the PL energy and the PL shift for the (311)B substrate orientation and for both $B \| z$ and $B \perp z$ can be summarized by the following evolution of the dots, shown schematically in Fig. 6. Without a GI [Fig. 6(a)], the dots are 
a)

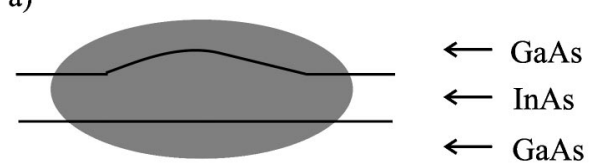

b)

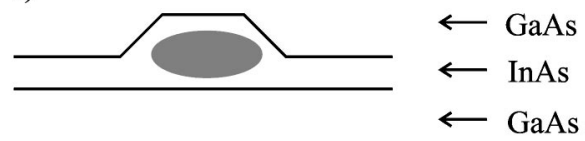

c)

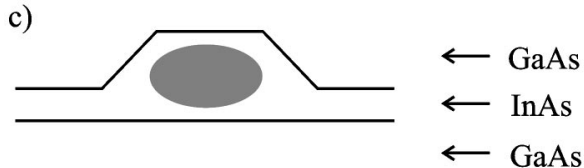

d)

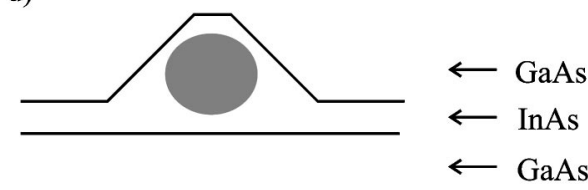

FIG. 6. Schematic representation of the evolution of the dots on the (311)B substrate. (a) shows the situation without growth interruption while (b), (c), and (d) show the ones after 40,80, and $120 \mathrm{~s}$ of growth interruption, respectively. The gray areas schematically represent the exciton wave function.

not yet completely developed and the wave functions of the electrons, and maybe the holes, are spilled out of the very thin wetting layer (with thickness fluctuations), resulting in a large $\Delta E_{\mathrm{CM}}$ for both field directions. After a GI of $40 \mathrm{~s}$ [Fig. 6(b)], the decrease in $\Delta E_{\mathrm{CM}}$ for both $B \| z$ and $B \perp z$ indicates that the charge carriers are more confined in both directions. The fluctuations in the wetting layer are transformed to more developed dots, which reduces not only the vertical confinement energy, giving a redshift of the PL energy, but also reduces the wave function extent. For a GI of $80 \mathrm{~s}$ [Fig. 6(c)], $\Delta E_{\mathrm{CM}}$ does not change for $B \|_{z}$ but it does for $B \perp z$. This indicates that the dots become higher while the diameter in the plane of the sample does not change significantly. This enlarging of the dots again corresponds to a redshift in the PL energy. Finally, after $120 \mathrm{~s}$ GI [Fig. 6(d)], there is an increase in $\Delta E_{\mathrm{CM}}$ for $B \perp z$ while for $B \|_{z} \Delta E_{\mathrm{CM}}$ again stays constant. So the vertical extent keeps increasing while the lateral extent of the wave functions stays unchanged. The diffusion of atoms causes the dots to become higher. One should thus expect a redshift of the PL energy, but instead a small blueshift is observed. This can be resolved when we no longer assume $\Delta E_{\mathrm{CM}}$ to be constant after $40 \mathrm{~s}$ GI but take into account the very small decrease in $\Delta E_{\mathrm{CM}}$ for $B \|_{z}$ [ [Fig. 4(b)] between 80 and $120 \mathrm{~s}$ GI, which results in a small decrease in the lateral extent of the wave functions. So the redshift of the PL energy, caused by the increasing height is compensated by a blueshift, caused by the decrease in lateral extent, resulting in an overall small blueshift of the PL energy. We note that a possible alternative explanation for the blueshift in PL energy could be the In desorbtion during the relatively long GI at relatively high temperature which leads

to a size decrease of the quantum dots. The transformation to fully developed dots between 0 and $40 \mathrm{~s}$ GI can also be seen in Fig. 4(c) where the product of $\mu / m_{0}$ and $\Delta E_{\mathrm{CM}}$ is plotted as function of the GI for $B \| z$. For the (311)B substrate, there is a big decrease in $\left(\mu / m_{0}\right) \times \Delta E_{\mathrm{CM}}$ between 0 and $40 \mathrm{~s}$, after which the product stays roughly constant.

Finally, bearing in mind the results of Ochoa et al., ${ }^{4}$ we speculate on the influence of the growth temperature on the formation of the dots. For a growth temperature of $460{ }^{\circ} \mathrm{C}$ on the (100) substrate, there is some diffusion of the adatoms, but long GIs are needed to form well-developed dots. Higher growth temperatures of $480{ }^{\circ} \mathrm{C}$ and $500{ }^{\circ} \mathrm{C}$ result in enough adatom diffusion to have efficient dot formation without GI. On the contrary, for the (311)B substrate orientation, no energy shift is observed at $460^{\circ} \mathrm{C}$. The combination of the high surface roughness and the low temperature is probably responsible for the fact that there is little diffusion of the adatoms, and this prohibits evolution during GI. A growth temperature of $480{ }^{\circ} \mathrm{C}$ enables the diffusion of adatoms, but due to the roughness of the surface, long GIs are necessary to form well-developed dots. When the growth temperature is raised to $500{ }^{\circ} \mathrm{C}$, there is sufficient diffusion of the adatoms and, just like for the (100) substrate, there is already efficient dot formation without GI.

\section{CONCLUSIONS}

A magnetophotoluminescence study of InAs QDs in GaAs grown by $\mathrm{MBE}$ at $480{ }^{\circ} \mathrm{C}$ revealed that the dots rapidly form an equilibrium morphology on the (100) substrate. On the contrary, for the (311)B substrate, a GI of $40 \mathrm{~s}$ is needed to transform the fluctuations in the wetting layer to more developed QDs. At longer GIs the dots become higher.

\section{ACKNOWLEDGMENTS}

This work was supported by the FWO-Vlaanderen and the Belgian IUAP programmes, the VIS 00/001 project of the K.U. Leuven, and the NANOMAT project of the EC Growth programme Contract No. G5RD-CT-2001-00545.

${ }^{1}$ K. Eberl, Phys. World 10, 47 (1997); D. Bimberg et al., Thin Solid Films 367, 235 (2000).

${ }^{2}$ J. Maes, M. Hayne, V. V. Moshchalkov, A. Patanè, M. Henini, L. Eaves, and P. C. Main, Appl. Phys. Lett. 81, 1480 (2002).

${ }^{3}$ A. Polimeni, A. Patanè, M. Henini, L. Eaves, P. C. Main, S. Sanguinetti, and M. Guzzi, J. Cryst. Growth 201-202, 276 (1999).

${ }^{4}$ D. Ochoa, A. Polimeni, M. Capizzi, A. Patanè, M. Henini, L. Eaves, and P. C. Main, J. Cryst. Growth 251, 192 (2003).

${ }^{5}$ R. Nötzel, T. Fukui, H. Hasegawa, J. Temmyo, and T. Tamamura, Appl. Phys. Lett. 65, 2854 (1994).

${ }^{6}$ P. B. Howes, J. L. Rawle, M. J. Everard, S. H. Baker, M. Henini, A. Patanè, and F. Pulizzi, IEEE conference NANO2003 12-14 August 2003, Proceedings of the Third IEEE Conference On Nanotechonology, 1-2, 130-133, 2003.

${ }^{7}$ M. Hayne, R. Provoost, M. K. Zundel, Y. M. Manz, K. Eberl, and V. V. Moshchalkov, Phys. Rev. B 62, 10324 (2000).

${ }^{8}$ J. Maes, M. Hayne, M. Henini, F. Pulizzi, A. Patanè, L. Eaves, and V. V. Moshchalkov, Physica B 346-347, 428 (2004).

${ }^{9}$ C. Pryor, Phys. Rev. B 57, 7192 (1998).

${ }^{10}$ D. M. Bruls et al., Appl. Phys. Lett. 81, 1708 (2002).

${ }^{11}$ J. H. Davies, The Physics of Low-Dimensional Semiconductors (Cambridge University Press, Cambridge, 1998). 